\title{
Possible half-metallic phase in bilayer graphene: Calculations based on mean-field theory applied to a two-layer Hubbard model
}

\author{
Jie Yuan, ${ }^{1}$ Dong-Hui Xu, ${ }^{2}$ Hao Wang, ${ }^{1,3}$ Yi Zhou, ${ }^{2}$ Jin-Hua Gao,,${ }^{4,1, *}$ and Fu-Chun Zhang ${ }^{1,2, \dagger}$ \\ ${ }^{1}$ Department of Physics, and Center of Theoretical and Computational Physics, The University of Hong Kong, Hong Kong, China \\ ${ }^{2}$ Department of Physics, Zhejiang University, Hangzhou, China \\ ${ }^{3}$ Department of Physics, South University of Science and Technology of China, Shenzhen, China \\ ${ }^{4}$ Department of Physics, Huazhong University of Science and Technology, Wuhan, Hubei, China
}

(Received 6 March 2013; published 21 November 2013)

\begin{abstract}
Charge neutral bilayer graphene has a gapped ground state, as transport experiments have demonstrated. One plausible ground state is the layered antiferromagnetic spin density wave (LAF) state, where the spins in the top and bottom layers have the same magnitude with opposite directions. We propose that lightly charged bilayer graphene in an electric field perpendicular to the graphene plane may be a half metal. We show this explicitly by using a mean-field theory on a two-layer Hubbard model.
\end{abstract}

DOI: 10.1103/PhysRevB.88.201109

PACS number(s): 73.22.Pr, 71.10.Fd, 71.30.+h, 73.21.Ac

Half metals are a class of materials in which electrons with one spin orientation are metallic and electrons with opposite spin orientation are insulating. ${ }^{1,2}$ In a half metal, the electric current can be fully spin polarized. This property is attractive in spintronics. ${ }^{3-5}$ The possibility of a graphene-based half metal is interesting for its potential application in electronic devices. Soon after the discovery of graphene, Son, Cohen, and Louie applied first principles calculations to propose a half-metallic phase in a zigzag graphene nanoribbon with external transverse electric fields. ${ }^{6}$ The predicted half metal in a graphene ribbon is still awaiting an experimental test. Very recently, there have been experiments to suggest that the ground state of bilayer graphene (BLG) at half filling (charge neutrality point) may be a layered antiferromagnetic spin density wave (LAF) state with a gap of about $2 \mathrm{meV}$. Here we propose that the slightly charged BLG in an electric field perpendicular to the graphene planes can be a half metal. We apply a mean-field theory on a two-layered Hubbard model, which is the simplest model to capture some basic physics in bilayer graphene. At half filling, we find the ground state to be a LAF state. The half-metallic phase is found at a slightly charged BLG under a transverse electric field within the LAF phase. Our prediction may be tested in the BLG device with double gates. ${ }^{7}$

We start with a brief summary of the recent works on the BLG. ${ }^{7-37}$ Theoretically, in the single electron band picture the BLG is a gapless semiconductor with parabolic valence and conduction bands touching at the high symmetry points $K$ and $K^{\prime}$. The gapless semiconducting state is unstable in the presence of electron interaction. Experimentally, there is clear evidence that the BLG at half filling has a gapped ground state. ${ }^{7,16-20}$ Velasco et al. ${ }^{7}$ have applied a perpendicular electric field on a high quality suspended BLG. The energy gap is found to decrease as the field increases and to close at a field $15 \mathrm{mV} \mathrm{nm}^{-1}$. Two of the most promising states consistent with the gapped ground states measured in transport experiments are the $\mathrm{LAF}^{26,27,33,38,39}$ and the quantum spin Hall states. ${ }^{26,27,33}$ It will be important and interesting to explore the possible experimental consequences of these states and determine the true ground state of the BLG. In this Rapid Communication, we predict a half-metallic phase in the LAF state of the BLG in the presence of an electric field in a slightly charged system.
Our result may be used to resolve the controversial issue of the ground state in a BLG, and should be of importance to the potential application of graphene in spintronics.

We consider a BLG system in an applied perpendicular electric field. The Hamiltonian is given by

$$
H=H_{0}+H_{U}+H_{p},
$$

where $H_{0}=H_{\text {intra }}+H_{\text {inter }}$ is the kinetic energy part, $H_{U}$ is the on-site Coulomb interaction, and $H_{p}$ describes the electric potential due to the applied electric field. The intralayer hopping is given by

$$
H_{\text {intra }}=-t \sum_{l\langle i j\rangle \sigma}\left[a_{l \sigma}^{\dagger}(i) b_{l \sigma}(j)+\text { H.c. }\right]+\mu \sum_{l i \sigma} n_{l \sigma}(i) .
$$

Here, $a_{l \sigma}\left(b_{l \sigma}\right)$ are the electron annihilation operators on sublattice $A(B), l=1,2$ for the bottom and top layers as illustrated in Fig. 1, and $\sigma=\uparrow, \downarrow$ and $i(j)$ denote the spin and site, respectively. \langle\rangle sums over all nearest neighbor sites. Here we only consider the nearest neighbor hopping for simplicity and expect that small remote hoppings will not change the basic physics. $\mu$ is the chemical potential. $\mu=0$ corresponds to the half filling, or the charge neutrality point. At $\mu>0$, the chemical potential crosses the conduction band. We consider an interlayer hopping $t_{\perp}$ between two sites $i$ and $i^{\prime}$ on top of each other,

$$
H_{\text {inter }}=t_{\perp} \sum_{\left\langle i i^{\prime}\right\rangle \sigma}\left[b_{1 \sigma}^{\dagger}(i) a_{2 \sigma}\left(i^{\prime}\right)+\text { H.c. }\right] .
$$

The Hubbard $U$ term is given by $H_{U}=U \sum_{l i}\left[n_{l \uparrow}(i)-\right.$ $1 / 2]\left[n_{l \downarrow}(i)-1 / 2\right]$. The effect of the external electric field $E_{0}$ is modeled by an electric potential $V$ between the two layers,

$$
H_{p}=\sum_{l i \sigma} V_{l} n_{l \sigma}(i)
$$

with $V_{l}=(-1)^{l} V / 2$, and $V$ is related to $E_{0}$ as below. Note that the electron charge density on the two layers may be redistributed in the presence of $E_{0} \cdot{ }^{13,14}$ Let $E$ be the electric field between the two layers, and assuming the graphene sheets to be infinitely large, we have $V=+e d_{0} E$, with $-e$ the electron charge and $d_{0}$ the interlayer distance. $E$ is related 

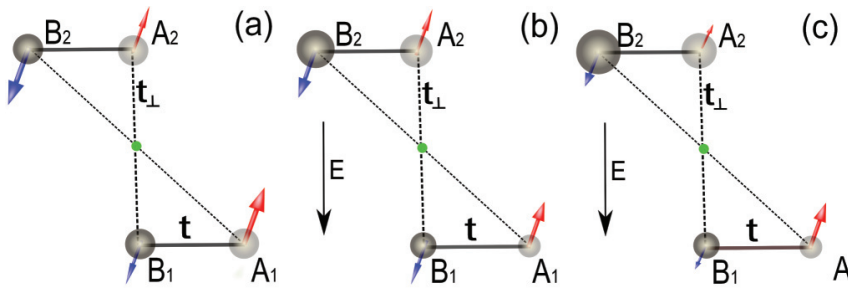

FIG. 1. (Color online) Schematic illustration of spin (arrows) and charge (circle) structures of the proposed layered antiferromagnetic state (LAF) in bilayer graphene. $A$ and $B$ indicate the two sublattices, and the subindices 1 and 2 are for bottom and top layers, respectively. (a) At half filling and at a perpendicular electric field $E=0$. Electron charges are uniform and the site spin polarization $P_{S}^{1 A}=-P_{S}^{2 B}$ and $P_{S}^{2 A}=-P_{S}^{1 B}$, with $P_{S}$ the average electron number difference between spin up and spin down on that site. (b) At half filling and finite $E$. The net charge is transferred from the bottom to the top layer, and the site spin polarizations have the same relation as in (a), with $E=0$. (c) Slightly electron doped graphene in a finite $E$. $P_{S}^{1 A}>\left|P_{S}^{2 B}\right|$ and $P_{S}^{2 A}>\left|P_{S}^{1 B}\right|$. The state has a net magnetic moment, and is a half metal.

to $E_{0}$ by

$$
E=E_{0}-2 \pi e\left(\rho_{2}-\rho_{1}\right),
$$

where the electron density in layer $l$ is given by $\rho_{l}=$ $\sum_{\sigma, i}\left\langle n_{l \sigma}(i)\right\rangle / S$, with $S$ the area of each layer, and $\langle Q\rangle$ is the average value of operator $Q$. In the above equation, we have assumed the dielectric constant for the BLG to be 1 , as suggested in the literature. ${ }^{13,40}$

We use a mean-field approximation for the Hubbard term, and solve the Hamiltonian self-consistently,

$$
H_{U}^{\mathrm{MF}}=U \sum_{l i \sigma}\left[\left\langle n_{l \sigma}(i)\right\rangle-1 / 2\right]\left[n_{l \bar{\sigma}}(i)-1 / 2\right] .
$$

There are four atoms in a unit cell and eight mean fields in our theory, $\left\langle n_{l \sigma}^{\eta}\right\rangle$, with $\eta=A$ or $B$ indicating the sublattice. In our calculations below, we use the intra- and interlayer hopping parameters $t=3.16 \mathrm{eV}$ and $t_{\perp}=0.381 \mathrm{eV}$, respectively. We use $d_{0}=0.334 \mathrm{~nm}$ and $2 \pi e^{2} d_{0} \approx 3 \times 10^{-11} \mathrm{meV} \mathrm{cm}^{2}$.

At $E_{0}=0$, our theory predicts a gapped LAF ground state at half filling for a finite value of repulsive Hubbard $U$. This result is consistent with previous theoretical works by using other methods such as renormalization group theory ${ }^{32,33}$ and the quantum Monte Carlo method. ${ }^{34}$ A gapped ground state is qualitatively consistent with a recent transport experiment. ${ }^{7}$ Note that the first principles calculation indicates that such a LAF state is stable in the presence of nonlocal Coulomb interaction and remote hopping. ${ }^{39}$ In Fig. 1(a), we schematically illustrate the spin and charge structures of the LAF state. The charge distribution is uniform and the spins are antiparallel to each other. Note that the antiparallel spins in the same layer have different magnitudes, which leads to net values of spins in each layer (spin down on the top layer and spin up on the bottom layer). The total spin or magnetism of the bilayer system is zero. In Fig. 2(a), we show the energy gap in the LAF state as a function of $U$. For $U \approx 6.64 \mathrm{eV}$, the mean-field theory gives a gap of $\epsilon_{g} \approx 2 \mathrm{meV}$, which is approximately the gap observed in the BLG. The low energy bands are shown in the inset of Fig. 2(a). Note that the value of
$U=6.64 \mathrm{eV}$ gives a ratio $U / t=2.10$ for the parameters in our calculations, and this ratio is smaller than the critical value of $U_{c} / t=2.23$ for the antiferromagnetism in the single layer Hubbard model obtained in the mean-field theory. Therefore, while the mean-field theory may not accurately estimate the gap or the critical value for $U,{ }^{41}$ our choice of the parameters here is self-consistent: It gives a nonmagnetic ground state of the single layer and a gap of $2 \mathrm{meV}$ for the bilayer. Coincidentally, the choice of the value of $U$ here is close to the value of $U \approx 6.2 \mathrm{eV}$ estimated within the mean-field theory to fit the experimentally observed energy gap in the $A B C$ stacking trilayer graphene. ${ }^{38,42}$ The inset in Fig. 2(a) shows the low energy dispersion near the $K^{\prime}$ point obtained in the mean-field theory for the bilayer Hubbard model for a typical value of $U$.

We proceed to discuss the effect of the electric field on the half-filled BLG. An electric field perpendicular to the graphene layer generates an electric potential difference $V$ between the top and bottom layers, which leads to electronic charge transfer from one to the other layer [bottom to top as illustrated in Fig. 1(b)]. At a weak electric field, the ground state remains to be LAF with neighboring spins antiparallel to each other. The charge imbalance changes the ground state spin distribution quantitatively in real space. More specifically, our calculations show that there is a charge transfer from sublattice $A$ in layer 1 to sublattice $B$ in layer 2, and a smaller amount of charge transfer from sublattice $B$ in layer 1 to sublattice $A$ in layer 2 (interlayer nearest neighboring sites). The low energy dispersion of the electron excitation becomes strongly spin dependent. This can be seen in Fig. 2(b), where we show the spin-dependent bands near the Fermi level for $V=1.4 \mathrm{meV}$. Both the lower energy valence and conduction bands are for spin-up electrons, and the spin-down electron bands are farther away from the Fermi level. Because of its semiconducting nature, all the valence bands are occupied and all the conduction bands are empty at zero temperature, and the ground state is spin nonpolarized. The spin-polarized low energy excitations can be detected in a spin-polarized transport experiment, and the gap measured by Velasco et $a l^{7}$ in the presence of an external electric field should be spin polarized, in agreement with previous work. ${ }^{27}$ The spin polarization of the excitation gap can be detected in transport measurements with ferromagnetic source and drain electrodes.

As the electric field increases, the gap for the spin-up electron bands continues to shrink. In the strong electric field limit, the ground state is a layered charge polarized (LCP) state, where the electron charge is imbalanced in the two layers and also on the two sublattices in the same layer. This state is not spin ordered, and is gapped, with the gap monotonically increasing as $V$ increases. In the LCP state, the Hubbard interaction $U$ becomes irrelevant. Our theory is similar to previous work on this state. ${ }^{27,28}$ Our mean-field theory suggests that the transition from the LAF to LCP state is first order, and the transition is within the gapped LAF state. As $V$ increases from 0 , the spin-up band gap decreases. The LAF state becomes unstable against the LCP at $V>V_{c}=1.8 \mathrm{meV}$. At $V=V_{c}$, the spin-up band gap of the LAF state remains finite although it is very small. As we can see from Fig. 2(c), the energy gap is spin dependent at $V<V_{c}$ in the LAF state, and becomes spin independent at 

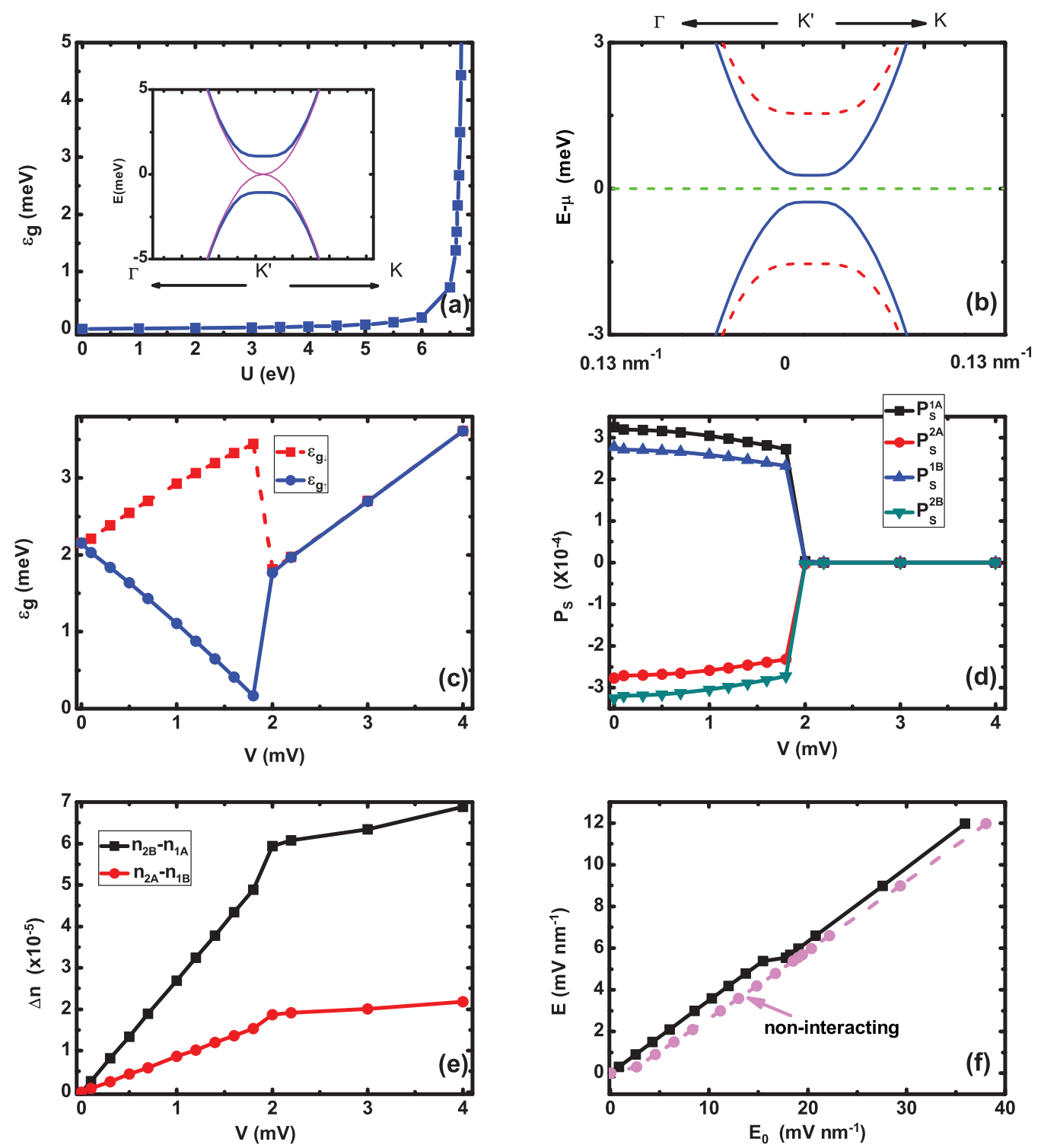

FIG. 2. (Color online) Bilayer graphene at half filling. (a) Energy gap of $H$ in Eq. (1) as a function of Hubbard $U$. Shown in inset are low energy bands (blue curves). The parameters are $U=6.64 \mathrm{eV}, t=3.16 \mathrm{eV}$, and $t_{\perp}=0.381 \mathrm{eV}$. The bands for $U=0$ are plotted for comparison (red curves). (b) Energy bands in a LAF state at the electric potential difference between the two layers $V=1.4 \mathrm{meV}$. Solid blue curves are for spin-up bands, and dashed red curves for spin-down bands. (c). Spin-resolved energy gap as a function of $V$. (d) Spin polarization on four distinct lattice sites. (e) Charge transfer as a function of interlayer electric potential $V$. In (d) and (e), superindices 1(2) and $A$ (B) are for layer indices and sublattices, respectively. In (c)-(e), the ground state is LAF at $V<V_{c}=1.8 \mathrm{mV}$, and the layered charge polarization state at $V>V_{c}$. (f) Electric field $E$ as a function of applied electric field $E_{0}$ (solid black line); also shown is $E$ vs $E_{0}$ for the noninteracting case $U=0$ (dashed pink line) for comparison.

$V>V_{c}$ in the LCP state. In Figs. 2(d) and 2(e), we show spin polarizations and charge distributions on each lattice site as functions of $V$. The spin polarization vanishes at $V>V_{c}$ in the LCP region, while the charge transfer has a kink at the transition point $V=V_{c}$ between the LAF and LCP states. We now briefly compare our electric-field-dependent energy gap with the transport experiment. ${ }^{7}$ In the transport experiment, the measured conductance is mapped onto the energy gap. As the electric field increases initially, the gap observed in the experiment decreases and closes, which is qualitatively consistent with our theory where the spin-up gap decreases to a tiny value. As the $E$ field further increases, the gap observed in the experiment saturates and then increases. The $E$-field dependence of the gap at large $E$ is also consistent with our theory, where we find the gap in the LCP state increases. The region around the transition point seems more complicated, and our theory predicts that a sudden increase in the gap value at the transition point $V_{c}$ from the LAF to LCP state for the transition is first order, as shown in Fig. 2(c). This implies a sudden drop in conductance at $V_{c}$. It will be interesting to test this in the experiment by measuring the conductance around the transition point more carefully. In Fig. 2(f), we show the screening effect and plot $E$ as a function $E_{0}$, according to Eq. (4). 


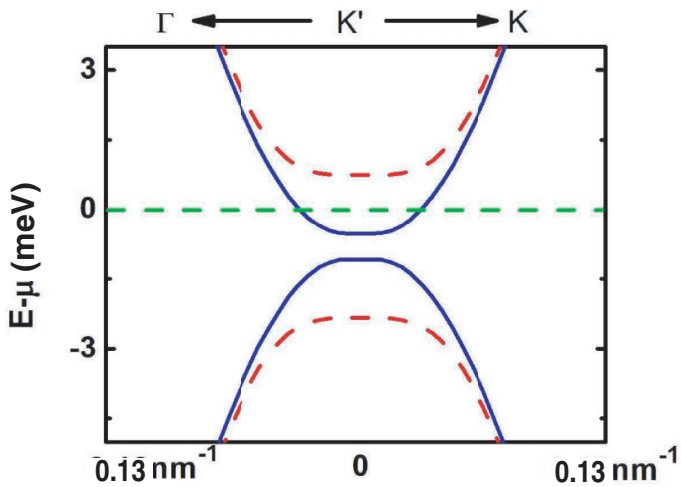

(a)

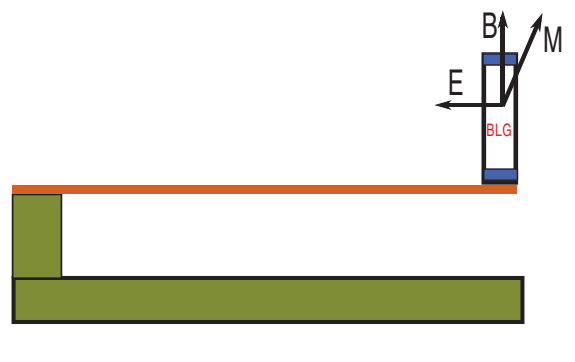

(b)

FIG. 3. (Color online) (a) Energy bands of the bilayer graphene in an interlayer electric potential $V=1.4 \mathrm{mV}$ at a charged graphene with electron density $\delta n \approx 2 \times 10^{10} \mathrm{~cm}^{-2}$. Solid lines are for spin up and dashed ones for spin down. Model parameters are the same as in Fig. 2 . (b) Schematics of probing magnetization by using a torque magnetometry experiment.

The most interesting case is at $\mu>0$ but small, where we have a metallic state in the background of LAF. The metallic state in a perpendicular electric field can be a half metal. The spin and charge structures of this case are illustrated in Fig. 1(c) and there is a net spin-up in the BLG. The case for $\mu<0$ can be obtained by particle-hole transformation and will not be discussed further. Experimentally, the shift on the chemical potential can be realized by tuning the gate voltage, which may be controlled independently together with the tuning of the electric field in a double gated BLG device. In Fig. 3(a), we plot the low energy bands for the BLG at the lightly charge density $\delta n \approx 2 \times 10^{10} \mathrm{~cm}^{-2}$. The other parameters are the same as in Fig. 2(b). The electron density is equivalent to $2.5 \times 10^{-6}$ electrons per carbon site on the BLG. As we can see from the figure, the spin-up conduction band is partially filled but the spin-down conduction band is completely empty. The system is a half metal with a full spin polarization in its carriers. The surface magnetization per area is $M=\delta n \times \mu_{G}=2 \times 10^{-4} g_{L} \mu_{B} / 2$ per $\mathrm{nm}^{2}$, with $\mu_{G}$ the magnetic moment of the graphene atom, $\mu_{B}$ the Bohr magneton, and $g_{L}$ the Lande $g$ factor for the graphene, which is about 2-2.5. The magnetization is tiny, but possibly detectable by using a torque magnetometry experiment, as schematically illustrated in Fig. 3(b). ${ }^{43}$ We have systematically studied $\mu$ or $\delta n$ dependence of the magnetization and the spin polarization of the BLG. The results are given in the Supplemental Material for a set of model parameters, which gives a more pronounced half-metallic state for a clear demonstration. ${ }^{44}$

In summary, we have applied a mean-field theory to study the LAF state in the BLG. Our theory predicts a half-metallic phase slightly away from the charge neutrality point in a transverse electric field, which may be realized in a double gated BLG. In the absence of electric field, the LAF state in BLG is invariant under a simultaneous transformation of time reversal and inversion operations, where the inversion is in respect to the midpoint of an interlayer bond (the green dot in Fig. 1), hence the total magnetism vanishes. The electric field breaks the inversion symmetry, opening the possibility for the global time reversal symmetry to be broken, which may be realized in the metallic LAF state for the lightly doped BLG. Finally, we argue that such a half-metallic phase should be found in the $N$-layer graphene with rhombohedral stacking ordering, where the LAF state has been proposed to be the ground state, as we reported recently. ${ }^{38}$ The LAF gap due to the electron interaction is larger for larger $N$, and saturates at a value of about $20 \mathrm{meV}$ around $N=9$. The half-metallic phase in multilayer graphene may be more robust than in the BLG, but perhaps more challenging to be realized in experiments. Theoretical work along this line is ongoing.

We thank W. Q. Chen for many useful discussions and $\mathrm{Lu} \mathrm{Li}$ for a helpful discussion on the possibility to use torque magnetometry to measure magnetization in layered systems. We acknowledge partial financial support from NSFC (Grant No. 11274269), HKSAR RGC (Grant No. HKU 701010 and AoE P-04/08). J.H.G. is supported by the National Natural Science Foundation of China (Project No. 11274129). D.H.X. and Y.Z. are supported by National Basic Research Program of China (973 Program, Grant No. 2011CBA00103), the NSFC (Grant No. 11074218), and the Fundamental Research Funds for the Central Universities in China.

\footnotetext{
*jinhua@hust.edu.cn

†fuchun@hku.hk

${ }^{1}$ R. A. de Groot, F. M. Mueller, P. G. van Engen, and K. H. J.

Buschow, Phys. Rev. Lett. 50, 2024 (1983).

${ }^{2}$ J. H. Park et al., Nature (London) 392, 794 (1998).
}

${ }^{3}$ S. A. Wolf et al., Science 294, 1488 (2001).

${ }^{4}$ S. Murakami, N. Nagaosa, and S. C. Zhang, Science 301, 1348 (2003).

${ }^{5}$ J. Sinova, D. Culcer, Q. Niu, N. A. Sinitsyn, T. Jungwirth, and A. H. MacDonald, Phys. Rev. Lett. 92, 126603 (2004). 
${ }^{6}$ Y. W. Son, M. L. Cohen, and S. G. Louie, Nature (London) 444, 347 (2006).

${ }^{7}$ J. Velasco, Jr., L. Jing, W. Bao, Y. Lee, P. Kratz, V. Aji, M. Bockrath, C. N. Lau, C. Varma, R. Stillwell, D. Smirnov, F. Zhang, J. Jung, and A. H. MacDonald, Nat. Nanotechnol. 7, 156 (2012).

${ }^{8}$ A. H. C. Neto, F. Guinea, N. M. R. Peres, K. S. Novoselov, and A. K. Geim, Rev. Mod. Phys. 81, 109 (2009).

${ }^{9}$ T. Ohta, A. Bostwick, T. Seyller, K. Horn, and E. Rotenberg, Science 313, 951 (2006).

${ }^{10}$ J. B. Oostinga, H. B. Heersche, X. Liu, A. F. Morpurgo, and L. M. K. Vanderspen, Nat. Mater. 7, 151 (2008).

${ }^{11}$ E. V. Castro, K. S. Novoselov, S. V. Morozov, N. M. R. Peres, J. M. B. Lopes dos Santos, J. Nilsson, F. Guinea, A. K. Geim, and A. H. Castro Neto, Phys. Rev. Lett. 99, 216802 (2007).

${ }^{12}$ Y. Zhang, T.-T. Tang, C. Girit, Z. Hao, M. C. Martin, A. Zettl, M. F. Crommie, Y. R. Shen, and F. Wang, Nature (London) 459, 820 (2009).

${ }^{13}$ E. McCann, Phys. Rev. B 74, 161403(R) (2006).

${ }^{14}$ H. Min, B. Sahu, S. K. Banerjee, and A. H. MacDonald, Phys. Rev. B 75, 155115 (2007).

${ }^{15}$ A. S. Mayorov, D. C. Elias, M. Mucha-Kruczynski, R. V. Gorbachev, T. Tudorovskiy, A. Zhukov, S. V. Morozov, M. I. Katsnelson, V. I. Fal'ko, A. K. Geim, and K. S. Novoselov, Science 333, 860 (2011).

${ }^{16}$ F. Freitag, M. Weiss, R. Maurand, J. Trbovic, and C. Schönenberger, Phys. Rev. B 87, 161402(R) (2013).

${ }^{17}$ R. T. Weitz, M. T. Allen, B. E. Feldman, J. Martin, and A. Yacoby, Science 330, 812 (2010).

${ }^{18}$ F. Freitag, J. Trbovic, M. Weiss, and C. Schönenberger, Phys. Rev. Lett. 108, 076602 (2012).

${ }^{19}$ A. Veligura, H. J. van Elferen, N. Tombros, J. C. Maan, U. Zeitler, and B. J. van Wees, Phys. Rev. B 85, 155412 (2012).

${ }^{20}$ W. Bao, J. Velasco, Jr., L. Jing, F. Zhang, B. Standley, D. Smirnov, M. Bockrath, A. H. MacDonald, and C. N. Lau, Proc. Natl. Acad. Sci. USA 109, 10802 (2012).

${ }^{21}$ J. Nilsson, A. H. Castro Neto, N. M. R. Peres, and F. Guinea, Phys. Rev. B 73, 214418 (2006).

${ }^{22}$ E. V. Castro, N. M. R. Peres, T. Stauber, and N. A. P. Silva, Phys. Rev. Lett. 100, 186803 (2008).
${ }^{23}$ H. Min, G. Borghi, M. Polini, and A. H. MacDonald, Phys. Rev. B 77, 041407(R) (2008).

${ }^{24}$ R. Nandkishore and L. Levitov, Phys. Rev. Lett. 104, 156803 (2010); Phys. Rev. B 82, 115124 (2010).

${ }^{25}$ J. Jung, F. Zhang, and A. H. MacDonald, Phys. Rev. B 83, 115408 (2011).

${ }^{26}$ F. Zhang, J. Jung, G. A. Fiete, Q. Niu, and A. H. MacDonald, Phys. Rev. Lett. 106, 156801 (2011).

${ }^{27}$ F. Zhang and A. H. MacDonald, Phys. Rev. Lett. 108, 186804 (2012).

${ }^{28}$ O. Vafek and K. Yang, Phys. Rev. B 81, 041401 (2010).

${ }^{29}$ Y. Lemonik, I. L. Aleiner, C. Toke, and V. I. Fal'ko, Phys. Rev. B 82, 201408 (2010); Y. Lemonik, I. L. Aleiner, and V. I. Fal'ko, ibid. 85, 245451 (2012)

${ }^{30}$ O. Vafek, Phys. Rev. B 82, 205106 (2010).

${ }^{31}$ M. Kharitonov, Phys. Rev. Lett. 109, 046803 (2012); Phys. Rev. B 86, 195435 (2012).

${ }^{32}$ R. E. Throckmorton and O. Vafek, Phys. Rev. B 86, 115447 (2012).

${ }^{33}$ M. M. Scherer, S. Uebelacker, and C. Honerkamp, Phys. Rev. B 85 , 235408 (2012)

${ }^{34}$ T. C. Lang, Z. Y. Meng, M. M. Scherer, S. Uebelacker, F. F. Assaad, A. Muramatsu, C. Honerkamp, and S. Wessel, Phys. Rev. Lett. 109, 126402 (2012)

${ }^{35}$ E. V. Gorbar, V. P. Gusynin, V. A. Miransky, and I. A. Shovkovy, Phys. Rev. B 85, 235460 (2012).

${ }^{36}$ X. Z. Yan and C. S. Ting, Phys. Rev. B 86, 235126 (2012).

${ }^{37}$ L. Zhu, V. Aji, and C. M. Varma, Phys. Rev. B 87, 035427 (2013).

${ }^{38}$ D.-H. Xu, J. Yuan, Z.-J. Yao, Y. Zhou, J.-H. Gao, and F.-C. Zhang, Phys. Rev. B 86, 201404 (2012).

${ }^{39}$ Y. Wang, H. Wang, J.-H. Gao, and F.-C. Zhang, Phys. Rev. B 87, 195413 (2013)

${ }^{40}$ F. Lonard and J. Tersoff, Appl. Phys. Lett. 81, 4835 (2002).

${ }^{41}$ S. Sorella and E. Tosatti, Europhys. Lett. 19, 699 (1992).

${ }^{42}$ M. M. Scherer, S. Uebelacker, D. D. Scherer, and C. Honerkamp, Phys. Rev. B 86, 155415 (2012).

${ }^{43}$ L. Li, C. Richter, J. Mannhart, and R. C. Ashoori, Nat. Phys. 7, 762 (2011).

${ }^{44}$ See Supplemental Material at http://link.aps.org/supplemental/ 10.1103/PhysRevB.88.201109 for $\mu$ and $\delta n$ dependence of magnetization and spin polarization of BLG away from half filling. 